\title{
Distinct ground state and activated state modes of firing in forebrain neurons
}

Daniel Levenstein ${ }^{1,2}$, Gabrielle Girardeau ${ }^{1,+}$, Jonathan Gornet ${ }^{2,+}$, Andres Grosmark ${ }^{1,+}$, Roman Huszár ${ }^{1,2,+}$, Adrien Peyrache ${ }^{1,+}$, Yuta Senzai ${ }^{1,+}$, Brendon Watson ${ }^{1,+}$, John Rinzel $^{2, *}$, György Buzsáki ${ }^{1,2,3,4, *}$

\section{Affiliations:}

${ }^{1}$ Neuroscience Institute, New York University, New York, NY 10016, USA

${ }^{2}$ Center for Neural Science, New York University, New York, NY 10003, USA

${ }^{3}$ Department of Neurology, New York University, New York, NY 10016, USA

Langone Medical Center, New York University, New York, NY 10016, USA

${ }^{4}$ Lead Contact

Present address:

(DL) Montreal Neurological Institute, McGill University, Montreal, QC H3A 2B4; Mila, Montreal, QC, H2S 3H1

(GG) Institut du Fer à moulin, UMR-S 1270, INSERM, Sorbonne Université, 75005 Paris.

(JG) McKelvey School of Engineering, Washington University in St. Louis. St. Louis, MO 63130

(AG) Department of Neuroscience, Columbia University, New York NY 10027

(AP) Montreal Neurological Institute, McGill University, Montreal, QC H3A 2B4

(YS) Department of Physiology, University of California, San Francisco, San Francisco, CA 94158, USA;

(BW) Department of Psychiatry, University of Michigan. Ann Arbor, MI

+ These authors contributed equally

*Correspondence: gyorgy.buzsaki@nyumc.org; rinzeljm@gmail.com

\section{ABSTRACT}

Neuronal firing patterns have significant spatiotemporal variety with no agreed upon theoretical framework. Using a combined experimental and modeling approach, we found that spike interval statistics can be described by discrete modes of activity. A "ground state" (GS) mode of low-rate spiking is universal among forebrain excitatory neurons and characterized by irregular spiking at a cell-specific rate. In contrast, "activated state" (AS) modes consist of spiking at characteristic timescales and regularity and are specific to neurons in a given region and brain state. We find that the majority of spiking is contributed by GS mode, while neurons can transiently switch to AS spiking in response to stimuli or in coordination with population activity patterns. We hypothesize that GS spiking serves to maintain a persistent backbone of neuronal activity while AS modes support communication functions. 


\section{INTRODUCTION}

A widely held view in neuroscience holds that the fundamental unit of neural computation is stochastic (Poisson-like) spiking following a continuously-varying rate $(1,2)$. It has been suggested that this irregular spiking arises as a consequence of inhibition balancing excitation in a "fluctuation-driven" regime (3-7) and allows neurons to maximize the efficiency of information transmission $(1,8)$. However, the irregular spiking view stands in contrast to the widely-observed constraint of spike times by oscillations (9) and biophysical properties (10), and the idea that neurons must form temporally-coordinated cell assemblies to effectively discharge their downstream target partners (11). Further, neuronal spike patterns vary between brain regions $(12,13)$, and depend on an animal's behavior, sensorium, and brain state (1416). Thus, the Poisson model alone does not adequately capture the wide range of observed spike-time statistics.

\section{RESULTS}

To search for an overarching characterization of neuronal spike patterns, we examined electrophysiological recordings from six regions of the rodent forebrain during waking and sleep (see Methods; Suppl. Fig. 1), and analyzed the distribution of log interspike intervals (ISIs) and their return maps $\left(I S I_{n}\right.$ vs ISI $\left.I_{n+1}\right)$ (Fig. 1A). Neurons in each region had qualitatively distinct ISI distributions, with characteristic "modes" of increased ISI density at specific timescales (Figure 1B) that were specific to brain state (NREM sleep vs WAKE/REM) and region (Suppl. Fig. 2).

\section{Ground state and activated state modes of interspike intervals}

Despite heterogeneity across brain regions, we noticed a universal pattern of ISIs in excitatory neurons (Fig. 1C,D, Suppl. Fig. 3). At long ISIs (low rate), distributions tended to show a single mode that was unique to each neuron. This low rate activity corresponded to an "on-diagonal" mode in the ISI return maps, and thus reflected sequential long ISIs rather than silent intervals between episodes of higher-rate spiking. In contrast to the neuron-specific lowest-rate mode, higher-rate modes were shared among neurons in the region. Each high-rate mode tended to have a distinct degree of regularity or irregularity, as measured by the ISI-conditioned coefficient of variation, CV2 (Suppl Fig. 4), with higher rate modes tending to have more regular spiking (CV2 < 1), compared to those with long ISIs (CV2 $\geq 1)$.

These observations prompted us to consider that the distinct modes of ISI distributions are the spiking correlate of distinct neuronal and network mechanisms (Fig. 1E). We hypothesized two main categories: a 'ground state' (GS) mode of irregular spiking at a cell-specific low rate, and a repertoire of 'activated state' (AS) modes common to neurons in a given brain region/state. 
A
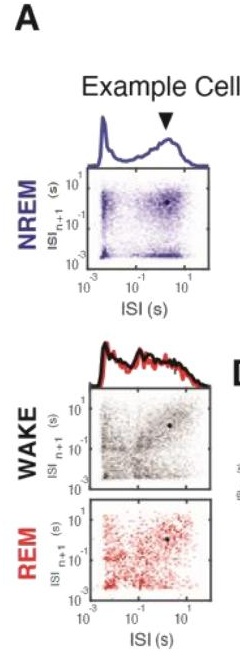

B
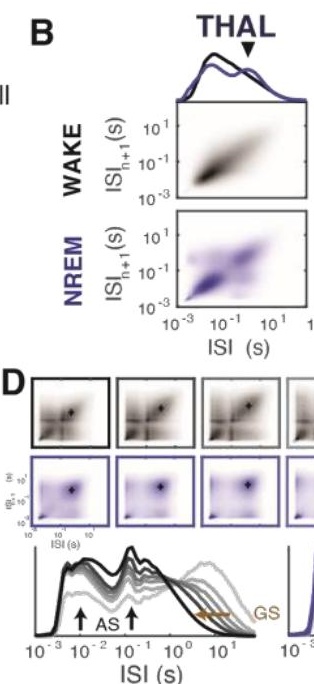

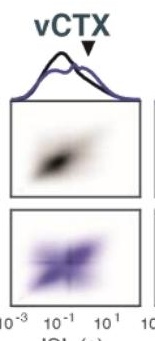

ISI (s)

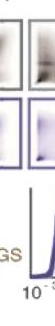

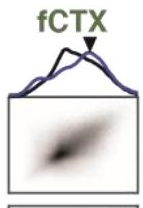

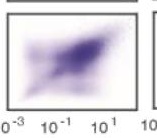

$1 \mathrm{SI}(\mathrm{s})$

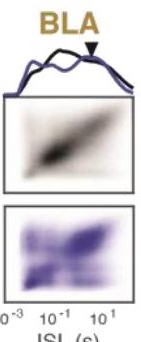

$I S I_{n}(s)$
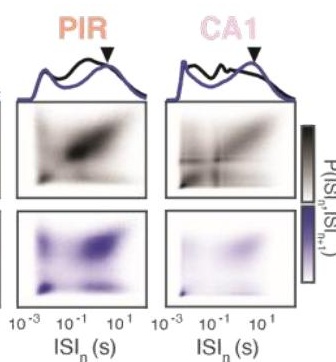

ACtive

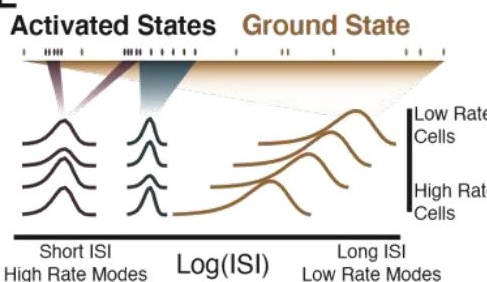

High Rate Modes Log(ISI) Low Rate Modes

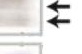

Figure 1: ISI distributions of forebrain excitatory neurons suggest a modal view of neural spiking with ground and activated states. A: logISI distribution and return map from an example CA1 cell during WAKE, REM, and NREM sleep. Ondiagonal clusters reflect sequential ISIs at a similar timescale, off-diagonal clusters reflect transitions between modes. B: Mean ISI distributions and return maps $\left(I_{n} I_{n}\right.$ vs $\left.I_{n+1}\right)$ from all putative excitatory cells in each region during WAKE and NREM sleep. Black triangles reflect mean firing rate over all cells in the region/state. Due to WAKE-REM similarity, only WAKE and NREM are shown - REM can be found in supplemental figures. C: logISI distributions for all CA1 pyramidal cells during WAKE and NREM sleep, sorted by mean firing rate. Black line indicates the mean ISI (1/mean rate) for each cell. (See Supp Fig 3 for comparable plots from all regions). D: Mean ISI distribution and return maps for CA1 cells divided into firing rate sextile groups. Location (i.e. rate) of the low rate mode (ground state, GS, brown) moves to shorter ISIs in higher firing rate groups, while pattern and timescale of higher rate modes (activated states, AS, black) are consistent across firing rate group. E: The ground state/activated state modal theory of neural activity.

To test our GS-AS hypothesis, we modeled the ISI distribution from each neuron with a mixture

model,

$$
\mathrm{P}_{i}[\log \mathrm{ISI}=\tau]=\sum_{m} a_{m, i} \psi_{m, i}(\tau)
$$

where $a_{m, i}$ is the weight, or fraction of spikes, from neuron $i$ in mode $m$, and each mode, $\psi_{m, i}(\tau)$, was taken to be a log-gamma distribution (17)(see Methods; Figure 2A-C, Suppl. Fig. 5). Fitting ISI distributions to this model allowed us to parameterize each mode by a characteristic rate (1/mean ISI) and variability (CV) which, when combined with the weights in each mode, revealed a "modal fingerprint" for the activity patterns of each neuron (Fig. 2B). In every brain region, two distinct clusters of spiking modes were seen: a low rate cluster of supra-Poisson spiking $(C V>1)$ that corresponded to the GS mode in each cell, and a cluster of sub-Poisson modes at higher spike rates with characteristic sub-clusters that were unique to each region/state (Fig. 2D). To further characterize the repertoire of AS modes in each region, we constrained the model such that the properties of each AS mode were shared across neurons in the same region/state (Fig 2D, Suppl. Fig. 6, Methods).

$$
\mathrm{P}_{i}[\log \mathrm{ISI}=\tau]=a_{G S, i} \psi_{G S, i}(\tau)+\sum_{m} a_{A S m, i} \psi_{m}(\tau)
$$


bioRxiv preprint doi: https://doi.org/10.1101/2021.09.20.461152; this version posted September 24, 2021. The copyright holder for this preprint (which was not certified by peer review) is the author/funder, who has granted bioRxiv a license to display the preprint in perpetuity. It is made available under aCC-BY-NC-ND 4.0 International license.
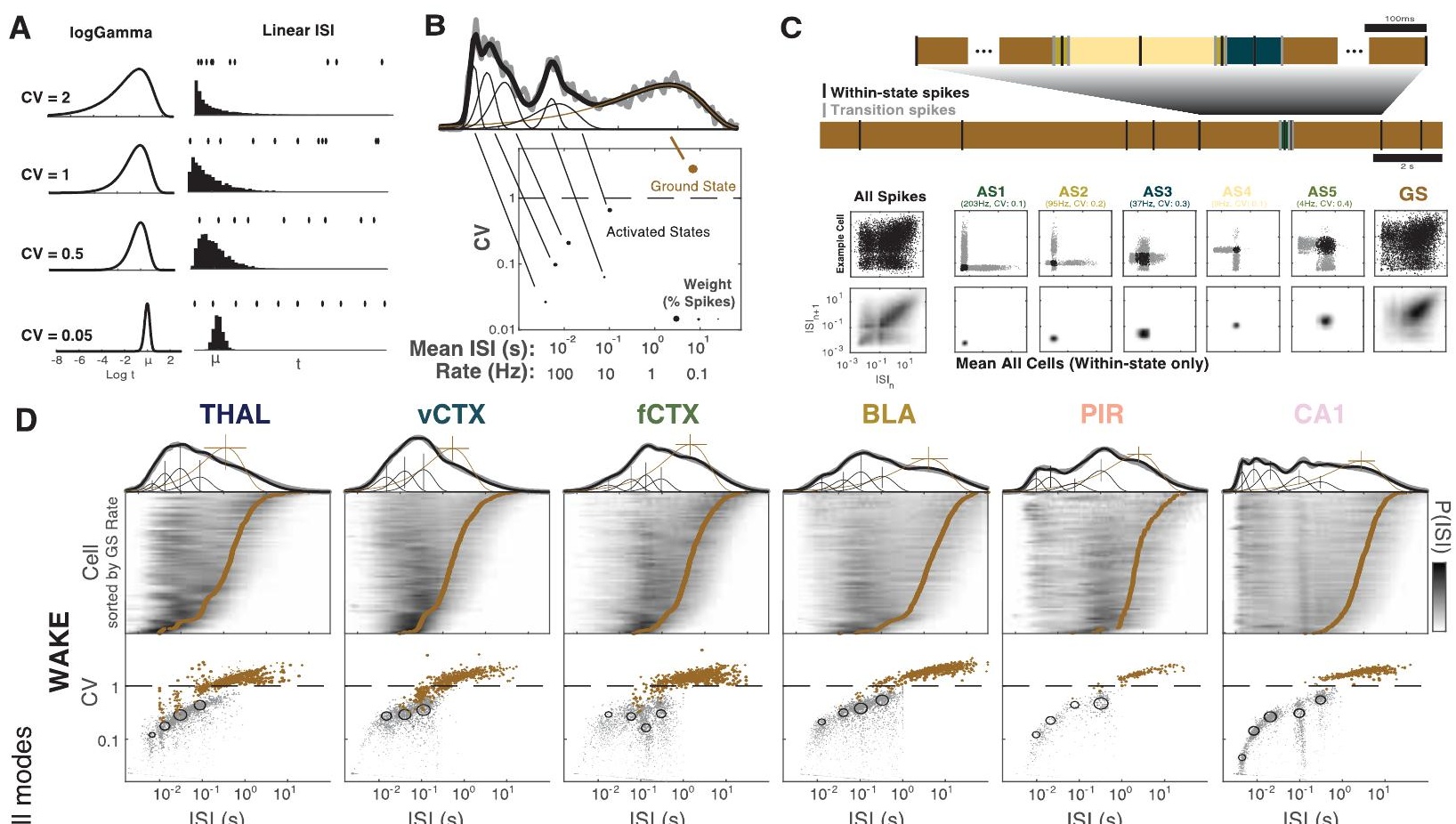
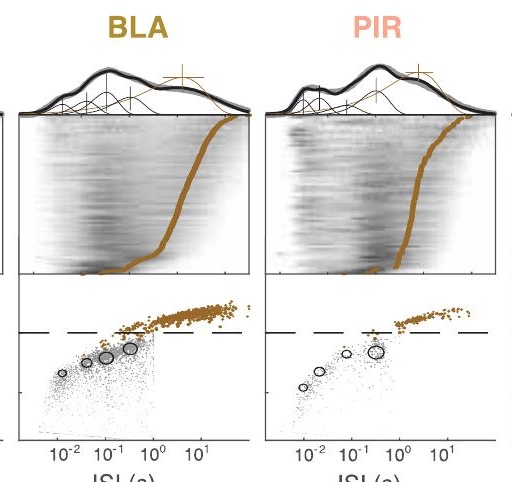

ISI (s)

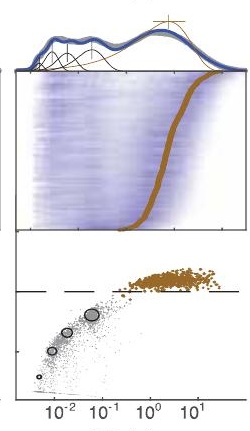

ISI (s)

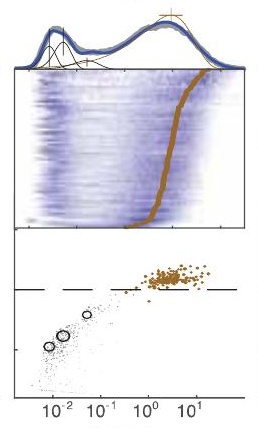

ISI (s)

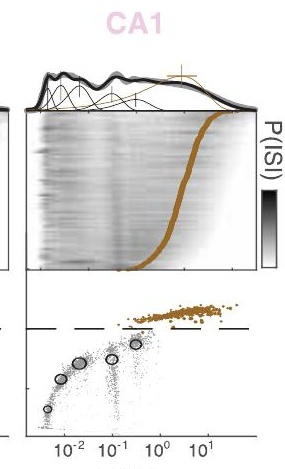

ISI (s)

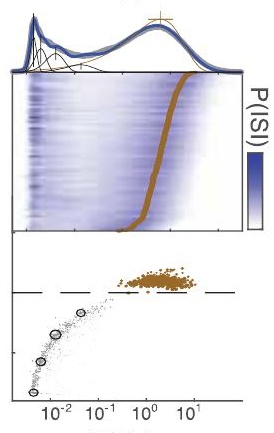

ISI (s)

Figure 2: Modal decomposition of ISI distributions and spike trains. A: (log)Gamma distribution as a model for (log)ISI modes. A logGamma distribution is characterized by a mean interval (i.e. a mean rate) and coefficient of variation (CV), which reflects the irregularity of spiking in that mode and can range from regular $(C V<<1)$, to poisson-like $(C V \sim 1)$, to supraPoisson $(C V>1)$ spiking. Four examples of spike trains and ISI distributions sampled from four logGamma distributions with a range of CVs are shown, each with mean ISI $=\mu$. B: Mixture of logGammas model fit for an example CA1 cell during WAKE. The logISI distribution for the cell is decomposed into a sum of logGamma distributions. Each mode is represented by its mean ISI (1/mean rate) and CV, and a weight, which reflects the proportion of spikes fired by the cell in that mode. The example cell has a ground state at $0.1 \mathrm{hz}$ with a CV=2, and 5 activated state modes at different rates/CVs. C: Hidden Markov Model for decomposing spike trains into modes. D: LogGamma mixture model fit for all cells in each region/state. Grey points correspond to individual cell fits. Open circles correspond to model fit with each AS mode constrained to have the same mean/CV over all cells ("shared" AS modes, see Methods), sized by the mean weight over all cells.

We then used a hidden Markov model (HMM) approach to assign each ISI to its most likely mode (Methods,

Fig. 2C).

Many AS modes corresponded to known network patterns in each region. For example, CA1 neurons during WAKE had an AS mode with regular spiking at $\sim 100 \mathrm{~ms}$ that was associated with theta oscillations ( $\sim \mathrm{Hz}$ ) (Supp Fig 7, (18)), and vCTX neurons had an AS mode of regular spiking at $250 \mathrm{~ms}$ which was associated with delta oscillations (Supp Fig 8, (19)). Further, neurons in each region had prominent AS modes at gamma timescales ( 10-40 ms) that were associated with local interneuron 
bioRxiv preprint doi: https://doi.org/10.1101/2021.09.20.461152; this version posted September 24, 2021. The copyright holder for this preprint (which was not certified by peer review) is the author/funder, who has granted bioRxiv a license to display the preprint in perpetuity. It is made available under aCC-BY-NC-ND 4.0 International license.

activation (20)(Supp Fig 9). Finally, a "burst" mode of regular spiking at $<10 \mathrm{~ms}$ was most prominent in the hippocampus during NREM sleep and likely reflects intracellular mechanisms (21)(Suppl. Fig. 10).

\section{Activated states of spiking}

To examine conditions that activate neurons out of the ground state, we examined ISI statistics during specific behaviors. The spike rate of a subset of hippocampal neurons increased at specific locations (22). When averaged across trials, spike rate varied smoothly with position. However, when we plotted ISIs as a function of position (Fig. 3A), this increased rate of spiking corresponded to the appearance of a discrete multi-modal cloud of 10-150ms ISIs, which was similar among all place cells (Fig. 3B-D). When we compared the in-field and out-field ISI distributions and return maps, we found that they differed in the relative occupancy in high- and low-rate modes, rather than reflecting a qualitative shift in the shape of the distribution (Fig. 3E). This change in mode occupancy reflected a decreased incidence of GS spiking and increased incidence of $30-100 \mathrm{~Hz}$ and $8 \mathrm{~Hz}$ AS modes within the place field, as identified by the HMM (Fig, 3B) and by increased weight in these modes when in- and out-field ISI distributions were fit separately with the mixture of gammas model (Fig. 3F).
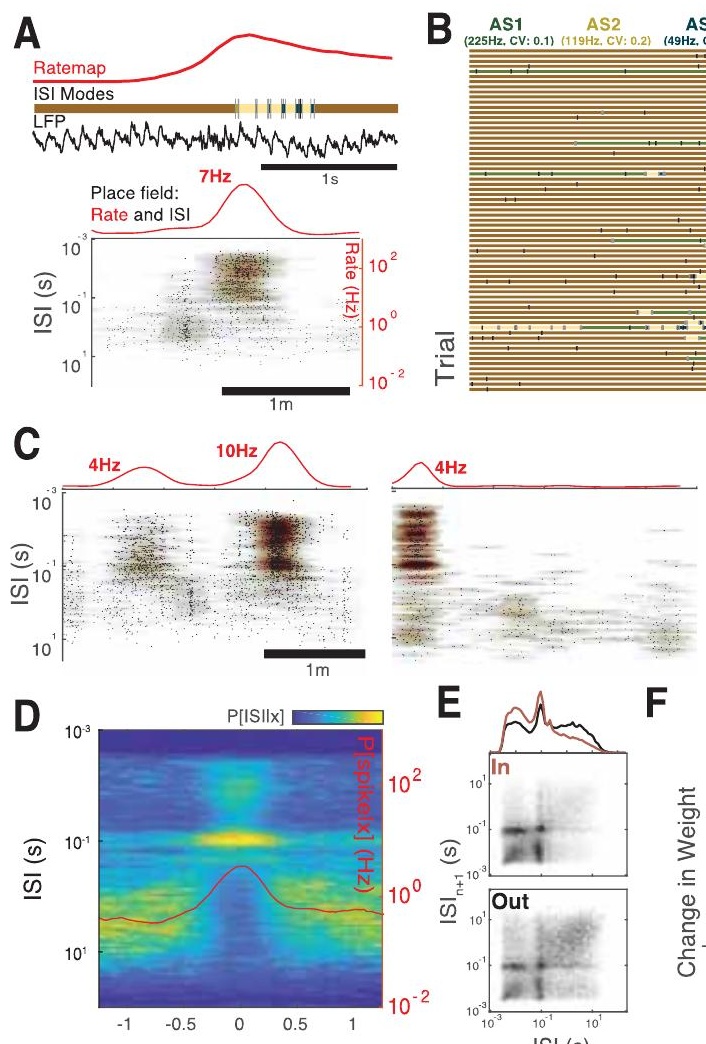

Position, relative to PF Peak (m)

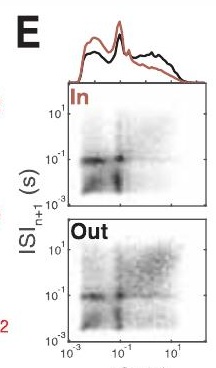

ISI (s)
$\mathbf{F}$

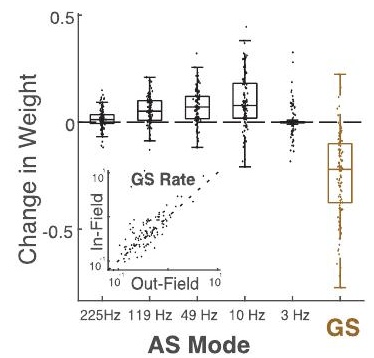

Figure 3: Place fields evoke theta/gamma activated states in CA1 cells. A: An example place cell. (Top) LFP, spikes, and spiking modes for a single traversal of the place field. (Bottom) adjacent ISIs from all spikes from all traversals, as a function of position. B: Trial-by-trial spiking modes, as classified by the HMM, for the example cell in A, aligned to entering the place field. C: ISIs as a function of position for three example place cells. Despite variation in peak rate, infield spikes have ISIs of similar timescales. D: Average position-conditional rate (P[spike | position]) and ISI distributions (P[ISI । position]) for CA1 place cells, centered on their place field peak. E: Mean in- and outfield ISI distributions and ISI return maps. Invs out-field ISI distributions do not differ in the general shape of the ISI distribution, but in the relative occupancy in activated state and ground state modes. F: Place fields increase occupancy of theta and gamma-timescale spiking modes, and decrease occupancy of GS modes. Change in weight for each mode between in- and out-field ISI distribution, as measured by the mixture of gamma model, for all CA1 place cells. (Inset) GS rate remains consistent in and out of the place field. 


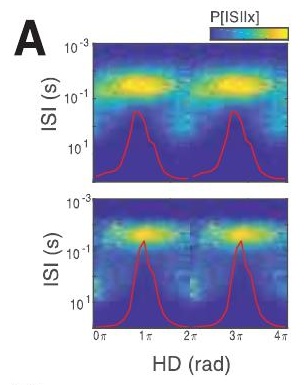

C

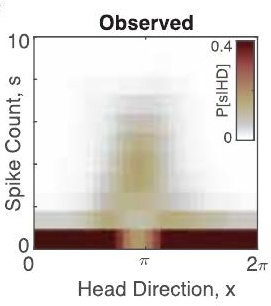

B
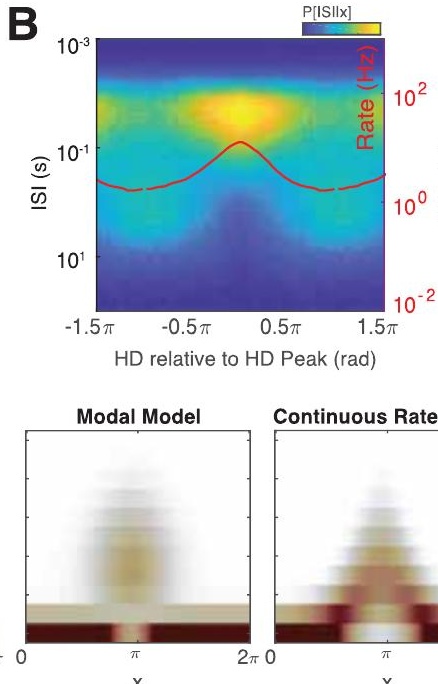

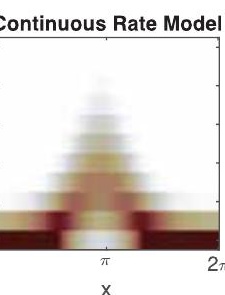

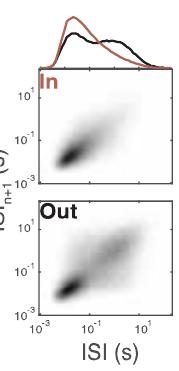

D

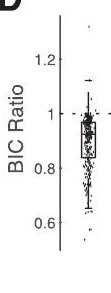

Figure 4: Sensory tuning reflects modal encoding. A: Rate (P[spike | HD], red line) and ISI distribution as a function of head direction for example head direction cells in the AD thalamus B: Average rate and conditional ISI for all HD cells, centered on their preferred head direction. While rate varies continuously with position and head direction, ISI distribution shows distinct modes of activity as a function of HD. (Right) Mean in- and out-field ISI distributions and ISI return maps. In- vs out-field ISI distributions do not differ in the general shape of the ISI distribution, but in the relative occupancy in activated state and ground state modes. C: (Left) Observed spike count given head direction for an example HD cell. (Right) Spike count given head direction for the modal and continuous model, with the best-fitting parameters for the example cell. D: Modal encoding model fits HD cell activity better than continuous rate encoding model. Bayesian information criterion $(\mathrm{BIC})$ ratio for model fits from each $\mathrm{HC}$ cell; lower ratio supports the modal model

To examine the generality of these findings, we treated thalamic head direction neurons (23) in a similar manner. Similar to $\mathrm{CA} 1$, we found that continuous variation in trial-averaged firing rate masked underlying discrete ISI modes. Timescale of ISIs in each mode was constant regardless of head direction, but occupancy was highest in the AS mode when the rat was gazing at the preferred direction of the neuron, whereas GS spikes dominated the less preferred directions (Fig. 4A, B). Because of the unimodal nature of the activated states in head direction neurons, we were able to directly compare a "continuous rate" encoding model, in which spike rate varied continuously with HD, with a discrete "modal" encoding model, in which the relative occupancy of a high rate (AS) mode and a low rate (GS) mode varied continuously with head direction (Methods, Fig. 4C, Suppl Fig. 11). In all HD cells, the modal model fit the observed spiking data better than the continuous rate model (Fig. 4D). This direct spike-count comparison between the modal model and a continuous-rate model also favors the modal version, without relying on analysis of ISIs.

\section{Ground State spiking reflects a default mode of balanced activity}

In contrast to region/state-specific AS modes, GS mode (Fig. 5A) was present in neurons in every brain region during all brain states. A neuron's firing rate was strongly correlated with its GS rate but less so with the occupancy of its AS modes or their rate (Fig. 5B,C), mainly because GS spikes represented the majority of spikes in most cells (Fig. 5E), and the vast majority of time was spent in the long-duration intervals between GS spikes (Suppl Fig. 12). A neuron's GS rate was conserved across WAKE and NREM states (Fig. 5F), and infraslow changes in firing rate (24) corresponded to changes in the occupancy of AS modes, rather than fluctuation of GS rate (Suppl. Fig. 13). Whereas AS mode spikes were coupled to particular frequency bands of the LFP, GS spikes were broadly coupled to the LFP at a wide range of frequencies (Suppl Fig. 14 (25)). 
A

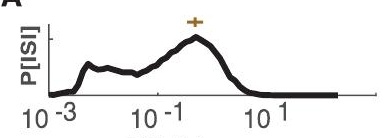

ISI (s)
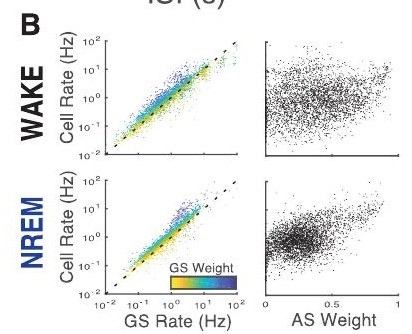

D
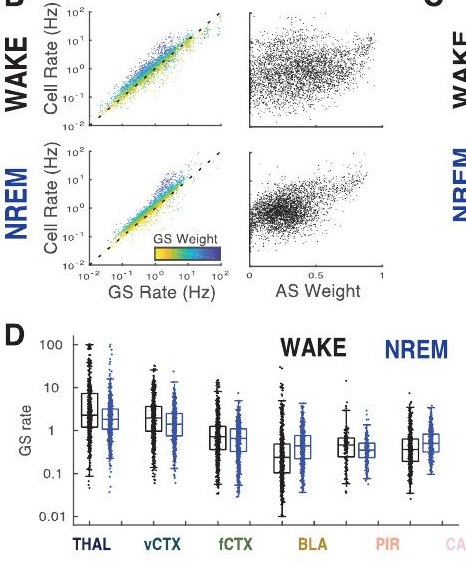

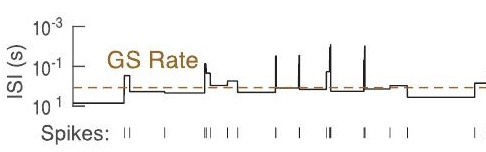

C

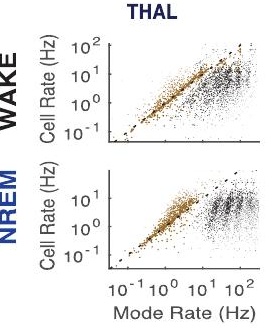

vCTX
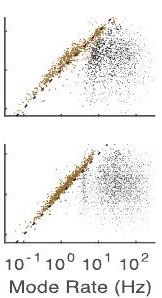

E

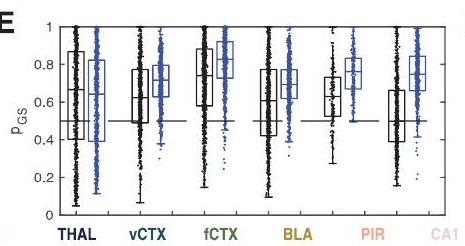

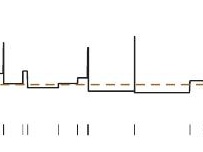

$\|$
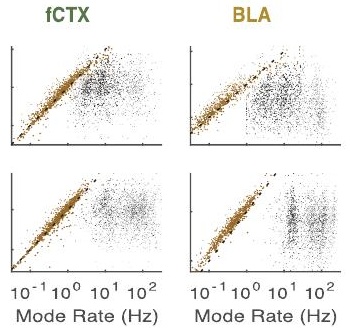

$\mathbf{F}$

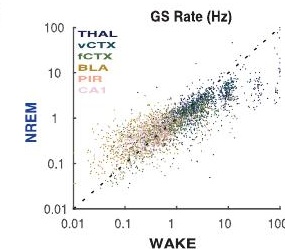

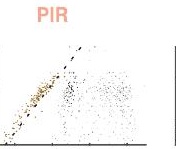
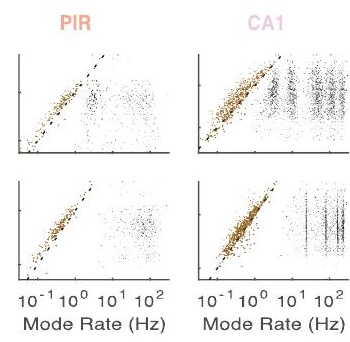

Mode Rate $(\mathrm{Hz})$

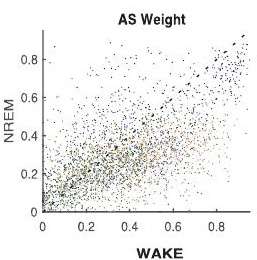

Figure 5: The Neuronal Ground State. A: Ground state activity in an example cell from CA1 - NREM. Left, ISI distribution. Cross indicates ground state rate. Right, sample spike train and ISI from the example cell. Note that the majority of time is spent in intervals around the GS rate (dashed line). B: Mean rate as a function of GS rate (left) and total AS weight (right) in all cells during WAKE and NREM. C: Mean rate as a function of modal rate from all modes from individual fit of all cells. Each cell can have up to 6 points in each plot, reflecting GS rate and the rate of up to 5 AS modes. In each region, rate of the GS mode is tightly correlated with mean rate, unlike AS modes. D: GS rate for all cells. $E$ : GS weight ( $p_{G S}$, fraction of spikes in the ground state) for all cells. Ground state spikes compose more than $50 \%$ of the ISI distribution in most cells from all regions/states. F: Ground state rate and total AS weight of all cells in WAKE and NREM.

Due to its irregular spiking ( $C V \geq 1$ ), we hypothesized that the GS mode arises from balanced inhibition and excitation. We tested this idea with a network model of integrate and fire neurons (Brunel 2000 ) in which each neuron used an inhibitory plasticity rule (26)(Fig 6A) to maintain a cell-specific target rate (Fig 6B, Suppl. Fig. 15, Methods). Under conditions of moderate excitatory (E-E) recurrent connectivity and near-threshold drive, the network showed a balanced regime of asynchronous activity (27), in which each excitatory neuron spiked irregularly at a cell-specific rate (i.e., GS-like activity, Fig. 6B,C). When we varied the strength of recurrent excitation or external drive, different activity patterns were induced in the network, including gamma-like oscillations (20), a heterogeneous asynchronous regime (28) and network bursts (Fig. 6D,E). Each of these regimes produced a characteristic pattern of AS modes in the ISI distributions, common to all neurons in the network and reflecting their engagement in collective activity. However, in each regime, GS activity was also preserved in the spiking of single neurons despite different collective dynamics.

\section{DISCUSSION}

Most previous research has focused on the problem of how neurons respond to sensory stimuli or induce motor outputs by changing a continuously varying rate of Poisson-like spiking. In contrast, our results suggest a framework in which single neurons engage in distinct spiking modes. One of these modes, the 


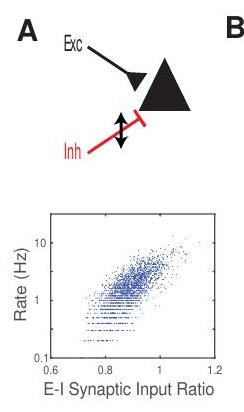

E
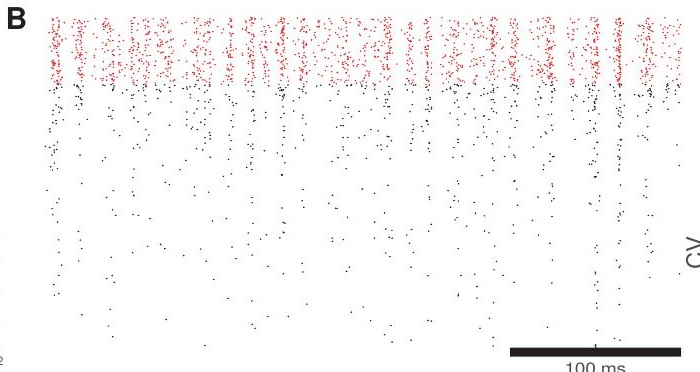

$\overrightarrow{0}$

$100 \mathrm{~ms}$

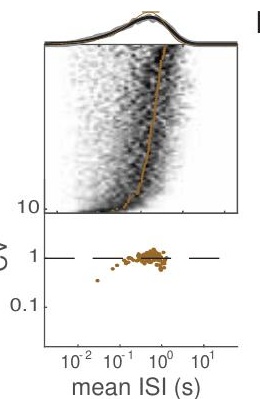

D

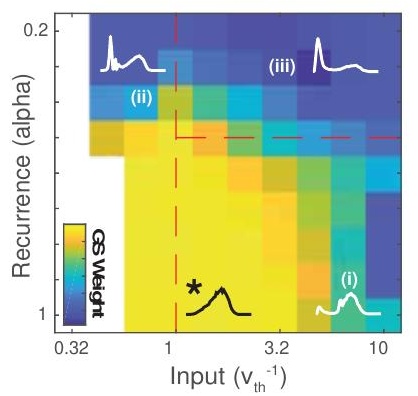

(ii)

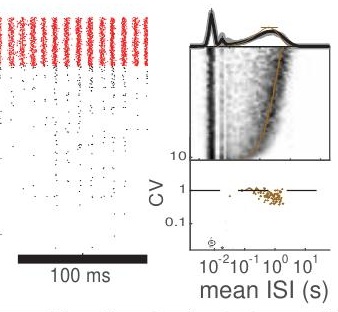

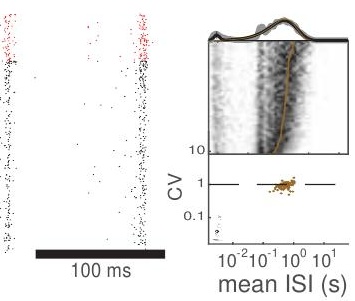

(iii)

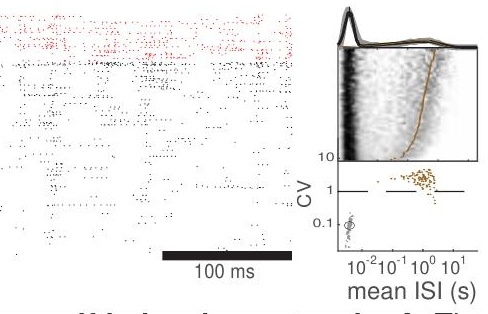

Figure 6: Ground/activated state activity is robustly produced by a heterogeneous self-balancing network. A: The heterogeneous self-balancing network. Each cell maintains spiking at a cell-autonomous rate by adjusting its incoming inhibitory synapses to balance excitatory inputs. B: GS rate reflects E/l synaptic input ratio. Firing rate is determined by the ratio of incoming excitatory and inhibitory synaptic weights in each cell. C: Ground state activity with irregular spiking in the heterogeneous selfbalancing network. Left: Spike raster from excitatory (black) and inhibitory (red) cells. Right: ISI distribution of a subset of cells in the network, sorted by GS rate, as identified by the mixture of gammas model. D: Mean GS weight over cells in the network, as determined by the mixture model, as a function of $\mathrm{E}-\mathrm{E}$ synaptic weights (recurrence) and the level of external drive. External drive and recurrence reduce GS weight by producing network activation patterns, each with associated AS modes (panel E), but GS mode is maintained. Recurrence is in units of $\mathrm{K}^{\wedge}(-$ alpha), where higher values of alpha signify lower excitatory weight. E: Simulated rasters (left) and ISI distributions fitted to the mixture of gamma model (right) for cells in self-balancing networks under conditions of strong drive (i), strong recurrence with low drive (ii), and strong recurrence (iii).

"ground state" (GS) mode is characterized by irregular spiking at a cell-specific low rate, and was found to be universal among neurons in all observed regions. In addition to the ground state, we found that neurons have a repertoire of higher-rate "activated state" (AS) modes. Each AS mode has a characteristic timescale and regularity and is common among neurons in the local population. We observed that specific AS modes were evoked in hippocampal place cells and thalamic head direction cells within their place fields and preferred head direction, respectively. We hypothesize that GS mode maintains the brain's internal dynamic, while AS modes serve communication functions.

We suggest that AS modes are the spiking correlates of various region-specific activity patterns, which emerge from network and cellular properties in a given region. In the self-balancing network model, a variety of different network activation patterns produced AS modes with spiking at similar timescale and regularity. These results suggest that the relationship between AS modes and network activity depends on the specific mechanisms that generate each AS mode. Regardless of generation mechanisms, the higherrate, more regular spiking of AS modes at particular timescales is ideal for effective transmission $(29,30)$ and multiplexing $(31,32)$ of functionally-distinct signals to downstream readers. For example, AS mode spiking might have mode-specific impact on postsynaptic cells (33) and networks (34), or activate intracellular processes in the spiking cell itself with mode-specific implications $(35,36)$.

In contrast to the higher-rate spiking of AS modes, slow irregular spikes are often considered as nonfunctional "noise", although the potentially beneficial role of noise has been acknowledged $(37,38)$. 
Because of the long ISIs of GS spikes, a target pyramidal neuron with short integration time constant $(<50$ $\mathrm{ms}$ ) will not be able to see the "rate" of GS spikes from each of its upstream partners. Yet, given that GS spikes represent the majority of spikes in the brain, their functional significance is expected to be important (Suppl. Fig. 16). The combined effect of ongoing GS activity might keep neurons in a near-threshold state from which they can respond quickly to relevant inputs $(38,39)$, and even solitary spikes can induce responses in target interneurons (33) or strongly-connected pyramidal cells (40). This sustained background activity from GS spikes may serve to maintain a homeostatically balanced backbone of neuronal activity in networks with vastly heterogeneous neurons (41-43). We hypothesize that the primary function of brain networks is to maintain their own dynamics and this is established by the GS mode of spiking. Finally, we propose that sequences or synchrony of GS spikes may offer an ongoing internal library, or reservoir, of activation patterns that are available to be selected and reinforced when associated with salient behaviors or stimuli (44-46).

\section{Author Contributions}

$\mathrm{DL}, \mathrm{GB}$ and JR conceived the experiments and framework, GG, AG, AP, YS, and BW performed the experiments, $\mathrm{DL}$ designed and executed all data analyses and computational modeling with assistance from $\mathrm{RH}$ on the HMM analysis and JG on the development of the spiking network, DL, GB and JR wrote the manuscript with contributions from other authors.

\section{Declarations of interest}

The authors declare no conflicting interests.

\section{Acknowledgements:}

The authors would like to thank Xiao-Jing Wang, Bijan Pesaran, Biyu He, Kenneth Harris, and Rachel Swanson for feedback on an early version of the manuscript. Supported by NIH MH122391, U19 NS104590, U19NS107616.

\section{Data availability}

The datasets and code used during the current study are available (data: https://crcns.org, code: https://github.com/buzsakilab/buzcode) 


\section{Citations}

1. M. N. Shadlen, W. T. Newsome, Noise, neural codes and cortical organization. Curr Opin Neurobiol. 4, 569-579 (1994).

2. M. N. Shadlen, W. T. Newsome, The Variable Discharge of Cortical Neurons: Implications for Connectivity, Computation, and Information Coding. J Neurosci. 18, 3870-3896 (1998).

3. G. L. Gerstein, B. Mandelbrot, Biophys J, in press, doi:10.1016/s0006-3495(64)86768-0.

4. C. van Vreeswijk, H. Sompolinsky, Science (New York, NY), in press.

5. B. Haider, A. Duque, A. R. Hasenstaub, D. A. McCormick, J Neurosci, in press, doi:10.1523/jneurosci.5297-05.2006.

6. M. Okun, I. Lampl, Nat Neurosci, in press, doi:10.1038/nn.2105.

7. A. Renart, J. de la Rocha, P. Bartho, L. Hollender, N. Parga, A. Reyes, K. D. Harris, Science, in press, doi:10.1126/science.1179850.

8. S. Denève, C. K. Machens, Nat Neurosci, in press, doi:10.1038/nn.4243.

9. G. Buzsáki, Rhythms of the Brain (2006).

10. C. Koch, Biophysics of Computation (1998), doi:10.1093/oso/9780195104912.001.0001.

11. D. O. Hebb, The Organization of Behavior. Wiley, New York, 378 (1949).

12. G. Maimon, J. A. Assad, Beyond Poisson: Increased Spike-Time Regularity across Primate Parietal Cortex. Neuron. 62, 426-440 (2009).

13. S. Shinomoto, H. Kim, T. Shimokawa, N. Matsuno, S. Funahashi, K. Shima, I. Fujita, H. Tamura, T. Doi, K. Kawano, N. Inaba, K. Fukushima, S. Kurkin, K. Kurata, M. Taira, K.-I. Tsutsui, H. Komatsu, T. Ogawa, K. Koida, J. Tanji, K. Toyama, Plos Comput Biol, in press, doi:10.1371/journal.pcbi.1000433.

14. B. O. Watson, D. Levenstein, J. P. Greene, J. N. Gelinas, G. Buzsáki, Neuron, in press, doi:10.1016/j.neuron.2016.03.036.

15. M. M. Churchland, B. M. Yu, J. P. Cunningham, L. P. Sugrue, M. R. Cohen, G. S. Corrado, W. T. Newsome, A. M. Clark, P. Hosseini, B. B. Scott, D. C. Bradley, M. A. Smith, A. Kohn, J. A. Movshon, K. M. Armstrong, T. Moore, S. W. Chang, L. H. Snyder, S. G. Lisberger, N. J. Priebe, I. M. Finn, D. Ferster, S. I. Ryu, G. Santhanam, M. Sahani, K. V. Shenoy, Nat Neurosci, in press, doi:10.1038/nn.2501.

16. A. Amarasingham, T.-L. Chen, S. Geman, M. T. Harrison, D. L. Sheinberg, Spike Count Reliability and the Poisson Hypothesis. J Neurosci. 26, 801-809 (2006).

17. S. Ostojic, Interspike interval distributions of spiking neurons driven by fluctuating inputs. $J$ Neurophysiol. 106, 361-373 (2011).

18. G. Buzsáki, L. L.-W. S., C. H. Vanderwolf, Cellular bases of hippocampal EEG in the behaving rat. Brain Res Rev. 6, 139-171 (1983).

19. Y. Senzai, A. Fernández-Ruiz, G. Buzsáki, in press (available at message:\%3CB0BD6BA6-E5514380-B5F0-A3DD2AD46FE2@gmail.com\%3E).

20. G. Buzsáki, X.-J. Wang, Mechanisms of Gamma Oscillations. Annu Rev Neurosci. 35, 203-225 (2012).

21. J. B. Ranck, Studies on single neurons in dorsal hippocampal formation and septum in unrestrained rats Part I. Behavioral correlates and firing repertoires. Exp Neurol. 41, 462-531 (1973).

22. J. O'Keefe, L. Nadel, The Hippocampus as a Cognitive Map (1978).

23. A. Peyrache, M. M. Lacroix, P. C. Petersen, G. Buzsáki, Nat Neurosci, in press, doi:10.1038/nn.3968.

24. M. Okun, N. A. Steinmetz, A. Lak, M. Dervinis, K. D. Harris, Distinct Structure of Cortical Population Activity on Fast and Infraslow Timescales. Cereb Cortex. 29, bhz023 (2019). 
25. B. O. Watson, M. Ding, G. Buzsáki, Eur J Neurosci, in press, doi:10.1111/ejn.13807.

26. T. P. Vogels, H. Sprekeler, F. Zenke, C. Clopath, W. Gerstner, Science, in press, doi:10.1126/science.1211095.

27. N. Brunel, Dynamics of Sparsely Connected Networks of Excitatory and Inhibitory Spiking Neurons. $J$ Comput Neurosci. 8, 183-208 (2000).

28. S. Ostojic, Nat Neurosci, in press, doi:10.1038/nn.3658.

29. J. S. Isaacson, M. Scanziani, Neuron, in press, doi:10.1016/j.neuron.2011.09.027.

30. M. Xue, B. V. Atallah, M. Scanziani, Nature, in press, doi:10.1038/nature13321.

31. D. Tingley, A. S. Alexander, L. K. Quinn, A. A. Chiba, D. Nitz, Multiplexed oscillations and phase rate coding in the basal forebrain. Sci Adv. 4, eaar3230 (2018).

32. R. Naud, H. Sprekeler, Sparse bursts optimize information transmission in a multiplexed neural code. Proc National Acad Sci. 115, 201720995 (2018).

33. D. F. English, S. McKenzie, T. Evans, K. Kim, E. Yoon, G. Buzsáki, Neuron, in press, doi:10.1016/j.neuron.2017.09.033.

34. A. Payeur, J. Guerguiev, F. Zenke, B. A. Richards, R. Naud, Burst-dependent synaptic plasticity can coordinate learning in hierarchical circuits. Nat Neurosci. 24, 1010-1019 (2021).

35. M. E. Larkum, Trends Neurosci, in press, doi:10.1016/j.tins.2012.11.006.

36. M. E. Larkum, J. J. Zhu, B. Sakmann, A new cellular mechanism for coupling inputs arriving at different cortical layers. Nature. 398, 18686 (1999).

37. E. M. Izhikevich, G. M. Edelman, Large-scale model of mammalian thalamocortical systems.

Proceedings of the National Academy of Sciences (2008) (available at http://www.pnas.org/content/105/9/3593.short).

38. J. Teramae, T. Fukai, Computational implications of lognormally distributed synaptic weights. $P$ leee. 102 (2014), doi:10.1109/jproc.2014.2306254.

39. K. Miura, Y. Tsubo, M. Okada, T. Fukai, J Neurosci, in press, doi:10.1523/jneurosci.2452-07.2007.

40. Y. Ikegaya, T. Sasaki, D. Ishikawa, N. Honma, K. Tao, N. Takahashi, G. Minamisawa, S. Ujita, N. Matsuki, Cereb Cortex, in press, doi:10.1093/cercor/bhs006.

41. K. Mizuseki, G. Buzsáki, Cell Reports, in press, doi:10.1016/j.celrep.2013.07.039.

42. D. Levenstein, B. O. Watson, J. Rinzel, G. Buzsáki, Curr Opin Neurobiol, in press, doi:10.1016/j.conb.2017.02.013.

43. A. Roxin, N. Brunel, D. Hansel, G. Mongillo, C. van Vreeswijk, J Neurosci, in press, doi:10.1523/jneurosci.1677-11.2011.

44. G. M. Edelman, Neural Darwinism: Selection and reentrant signaling in higher brain function. Neuron. 10, 115-125 (1993).

45. W. Maass, T. Natschläger, H. Markram, Neural Comput, in press, doi:10.1162/089976602760407955.

46. G. Buzsáki, The Brain from Inside Out (2019), doi:10.1093/oso/9780190905385.001.0001. 